\title{
Paper
}

\section{Electromagnetic Scattering of Inhomogeneous Plane Wave by Ensemble of Cylinders}

\author{
Lorenzo Dinia $^{1}$, Fabio Mangini ${ }^{2}$, and Fabrizio Frezza ${ }^{1}$ \\ ${ }^{1}$ Department of Information Engineering (DII), University of Brescia, Brescia, Italy \\ ${ }^{2}$ Department of Information Engineering, Electronics and Telecommunications (DIET), "Sapienza" University of Rome, Rome, Italy
}

https://doi.org/10.26636/jtit.2020.138519

\begin{abstract}
The interaction between an ensemble of cylinders and an inhomogeneous plane wave is introduced and is determined, in the present paper, through a rigorous theoretical approach. Scattered electromagnetic field generated by an indefinite number of infinite circular cylinders is analyzed by the application of the generalized vector cylinder harmonics (VCH) expansion. The exact mathematical model relied upon to represent this scenario considers the so-called complex-angle formalism reaching a superposition of vectorial cylindrical-harmonics and Foldy-Lax Multiple scattering equations (FLMSE) to account for the multiscattering process between the cylinders. The method was validated by comparing the numerical results obtained with the use of the finite element method and a homemade Matlab code.
\end{abstract}

Keywords-electromagnetic scattering, inhomogeneous wave dispersion, lossy media, multi-cylinders scattering, vectorial cylindrical-harmonics.

\section{Introduction}

In the last decades, researchers were focusing on solving Maxwell's equations in order to determine the field scattered by a single or by multiple objects with a specific geometry. Geometrically, electromagnetic scattering has already been analyzed in a thorough manner. In fact, spherical, spheroidal, cylindrical, conical, and ellipsoidal objects may be found in literature, in several works devoted to canonical scattering [1]-[6]. In addition to the cases mentioned above, many other complex scenarios have been examined, such as spheres, cylinders, and axially symmetric objects [7]-[13]. Ensembles of different configurations of scatterers are also considered [14]-[17]. Moreover, different physical and chemical characteristics of materials constituting the scatterer have been analyzed [18]-[21]. Over the years, the problem of describing the interaction between the electromagnetic field and a set of cylinders has led to the development of numerous exact theoretical models that are increasingly simplified [8]-[10].

In this paper, an accurate method for showing an elliptically polarized inhomogeneous plane wave as an expan- sion of vector cylindrical-harmonics ( $\mathrm{VCH})$ is presented. Moreover, in order to analyze the multi-scattering process, the so-called T-matrix approach [22], [23] is used, applying Foldy-Lax multiple scattering equations (FLMSEs) [24], [25] to ensure continuity of tangential components of the electromagnetic fields on the surface of each scatterer. The general representation of an electromagnetic wave as an inhomogeneous wave has continuously attracted a lot of researchers' interest. An electromagnetic wave that propagates in a lossy medium is represented by a complex wave vector with two components: a phase vector and an attenuation vector. A completely lossless medium is an ideal scenario that does not exist in nature. In fact, a wave typically propagates in a lossy medium.

In 1987, the first study related to an inhomogeneous elliptically polarized plane wave was presented by Ivlev, describing the propagation in its elemental structure and investigating the energy fallout as well [26], [27]. The first application proposed subsequently by Ivlev was on an indefinite cylinder. The Adler-Chu-Fano formulation with phase and attenuation vectors [28] was adopted in these studies, leading to meaningful and elegant results with a high degree of complexity.

The present study shows how the representation of the incident field as VCHs superposition could be reached with much greater simplicity through the use of the complexangle [29] formulation. Also, this approach will be generalized for a scenario involving scattering caused by a cylinder immersed in a lossy medium. Our goal is to use these simplified models and to introduce a concept of losses in the propagation medium in a minimally invasive way. The results will be suitable for updating models, codes, and software presented in the literature to describe real situations with a minimal impact on the code itself. The paper provides numerical comparisons of different phenomena involving cylindrical vector waves. In addition, the study focuses on the analysis of an ensemble of infinite lossy cylinders immersed in a lossy medium, and on its results. A multi-paradigm numerical computing environment (Matlab) was used to implement the various formulations, while the proper model was simulated with the 
use of Comsol Multiphysics - commercial software based on FEM.

The article is divided into 4 parts. Section 2 introduces two principal formalisms required to properly represent an inhomogeneous wave and illustrates the formulas needed to switch between both of them. Subsequently, theoretical aspects are described in order to obtain a representation of an inhomogeneous elliptically polarized electric field as a superposition of VCHs. Section 3 shows numerical validations concerning results obtained in Matlab and the ones generated with the use of Comsol Multiphysics. In the same section, new results are also presented for the scattering of an elliptically polarized plane wave at oblique incidence from $n$-cylinders in a lossy medium. Finally, in Section 4, conclusions are given, along with potential future developments.

\section{Theoretical Approach}

From literature, two formalisms are known to be used for representing an inhomogeneous wave propagating in a lossy medium. The first one, characterized by better features as well, is the formalism known as Adler-Chu-Fano formulation. Its propagation vector has a complex nature with $\boldsymbol{k}_{i}=\boldsymbol{\beta}_{i}+i \boldsymbol{\alpha}_{i}$ represented by phase and attenuation vectors $\boldsymbol{\beta}_{i}, \boldsymbol{\alpha}_{i} \in \Re$, respectively. The other one has a complex propagation vector as well, represented by the superposition of real and imaginary parts $\boldsymbol{k}_{i}=k_{\mathbf{R}}+i k_{\mathbf{I}}$. This vector forms a complex angle with the axis of the Cartesian reference system $\bar{\vartheta}_{i}=\vartheta_{\mathbf{R}}+i \vartheta_{\mathbf{I}}$ [29], see Fig. 1. The symbol $\bar{\vartheta}$ was used to underline the complex nature of the angle.

This study shows that a field expressed as a superposition of basic cylindrical waves through the use of the complexangle formalism may be represented with relative simplicity. The following wave, in which vectors $\alpha_{i}$ and $\beta_{i}$ are creating angles $\zeta_{i}$ and $\eta_{i}$ in relation to the $z$ axis, are also placed on the same plane passing through the $z$ axis, and are forming a real angle $\varphi$ with the $x$ axis (see Fig. 1). In this particular case, the following relations exist between the two formalisms [29]:

$$
\begin{aligned}
& \cos \vartheta_{\mathbf{R}}=\frac{k_{\mathbf{R}} \beta \cos \xi+k_{\mathbf{I}} \alpha \cos \eta}{\sqrt{k_{\mathbf{R}}^{2} \beta^{2}-k_{\mathbf{I}}^{2} \alpha^{2}+2\left(k_{\mathbf{R}} k_{\mathbf{I}}\right)^{2}}}, \\
& \sin \vartheta_{\mathbf{R}}=\frac{k_{\mathbf{R}} \beta \sin \xi+k_{\mathbf{I}} \alpha \sin \eta}{\sqrt{k_{\mathbf{R}}^{2} \beta^{2}-k_{\mathbf{I}}^{2} \alpha^{2}+2\left(k_{\mathbf{R}} k_{\mathbf{I}}\right)^{2}}}, \\
& \vartheta_{\mathbf{I}}=\frac{1}{2} \operatorname{atanh}\left(\frac{2 \beta \alpha}{k^{2}}\right)
\end{aligned}
$$

where $\eta$ and $\xi$ are the angles that the vectors $\boldsymbol{\alpha}$ and $\boldsymbol{\beta}$, respectively, form with the $z$ axis. Equations (1) and (2) play a fundamental role in assigning a value to $\vartheta_{\mathbf{R}}$ avoiding its indetermination. Plane $\varphi=0$ was considered due to its simplicity, although the following considerations can be easily expanded to each plane with $\varphi \neq 0$.

The solution of the scalar Helmholtz equation provides the following scalar formula [30]-[36]:

$$
\psi_{m}=A \mathrm{e}^{i m \varphi} Z_{m}\left(k_{\rho} \rho\right) \mathrm{e}^{i k_{z} z-i \omega t},
$$

where $\rho, \phi, z$ are three variables independent of the cylindrical coordinate system, $A$ is a complex constant, while $k_{\rho}$ and $k_{z}$ are the projections of the propagation vector on the plane $z=0$ and the on the $z$ axis, respectively. The last two components are defined as shown below:

$$
k_{\rho}^{2}+k_{z}^{2}=k^{2}
$$

with $k_{x}=k_{\rho} \cos \varphi$ and $k_{y}=k_{\rho} \sin \varphi$ being projections of the transversal vector $k_{\rho}$ on the plane $z=0$. The function $Z_{m}\left(k_{\rho} \rho\right)$ describes the first, second, third, and fourth Bessel functions as $J_{m}\left(k_{\rho} \rho\right), Y_{m}\left(k_{\rho} \rho\right), H_{m}^{(1)}\left(k_{\rho} \rho\right)$, and $H_{m}^{(2)}\left(k_{\rho} \rho\right)$, respectively. Hence, the harmonic vector is characterized as [31]-[36]:

$$
\mathbf{M}=\nabla \times\left(\hat{\mathbf{z}}_{\mathbf{0}} \psi\right), \quad \mathbf{N}=\frac{1}{k} \nabla \times \mathbf{M}
$$
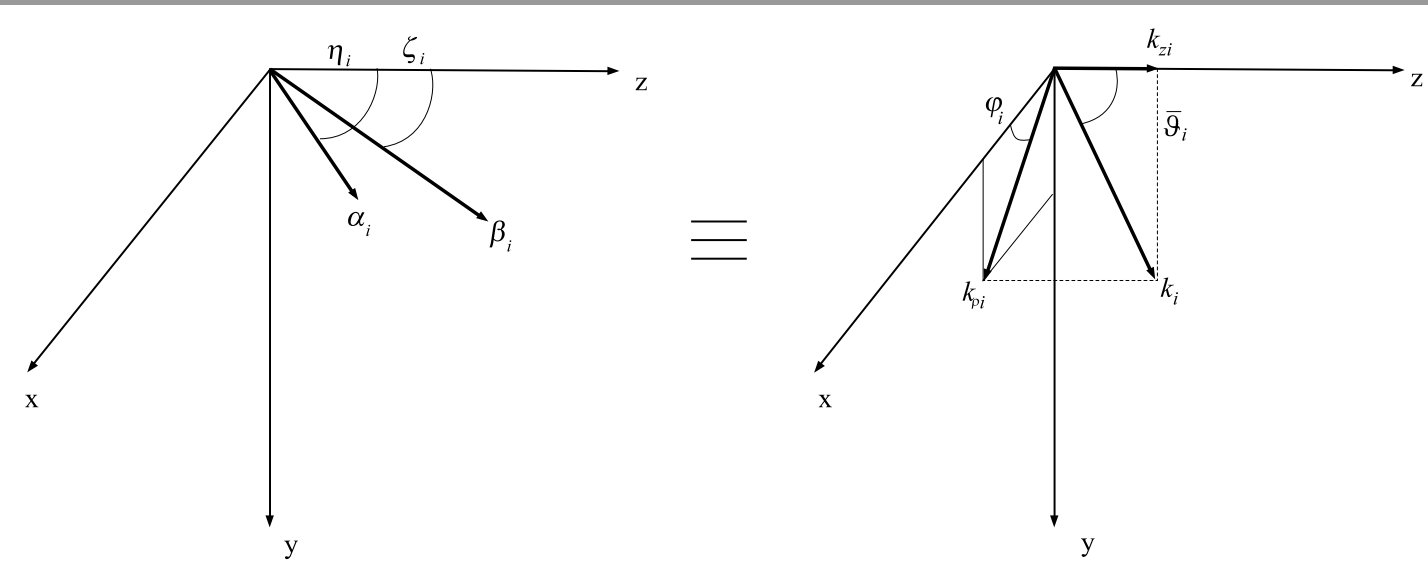

Fig. 1. The figure on the left represents the complex wave vector of an inhomogeneous plane wave with phase and attenuation vectors. The figure on the right represents the same vectors with the complex-angle formulation. 
It is always possible to define electric and the magnetic fields as a superposition of these vectorial functions:

$$
\begin{aligned}
& \mathbf{E}=\sum_{m=-\infty}^{+\infty}\left(a_{m} \mathbf{M}_{m}+b_{m} \mathbf{N}_{m}\right) \\
& \mathbf{H}=\frac{k}{i \omega \mu} \sum_{m=-\infty}^{+\infty}\left(a_{m} \mathbf{N}_{m}+b_{n} \mathbf{M}_{m}\right) .
\end{aligned}
$$

Let us consider a simple inhomogeneous plane wave, using the formalism presented for the first time by Frezza et al. [36], [37]. Any obliquely polarized elliptical field, with respect to the surface of a cylinder, can be represented as a linear combination of two components, one vertical $\left(\mathbf{v}_{0}\right)$ and one horizontal $\left(\mathbf{h}_{0}\right)$, each multiplied by its polarization coefficient $\left(E_{v i}\right.$ and $E_{h i}$, respectively):

$$
\begin{aligned}
& \mathbf{E}(\mathbf{r})=\left[E_{v i} \mathbf{v}_{0}\left(\bar{\vartheta}_{i}, \varphi_{i}\right)+E_{h i} \mathbf{h}_{0}\left(\bar{\vartheta}_{i}, \varphi_{i}\right)\right] \mathrm{e}^{i \mathbf{k} \cdot \mathbf{r}} \\
& =\sum_{m=-\infty}^{+\infty}\left[a_{m} \mathbf{M}_{m}\left(k^{*} \mathbf{r}\right)+b_{m} \mathbf{N}_{m}\left(k^{*} \mathbf{r}\right)\right]
\end{aligned}
$$

imposing the following definitions [36]:

$$
\begin{aligned}
& a_{m}=\frac{E_{h i}}{k_{\rho}}(-i)^{m-1} \mathrm{e}^{-i m \varphi_{i}}, \\
& b_{m}=-\frac{E_{v i}}{k_{\rho}}(-i)^{m} \mathrm{e}^{-i m \varphi_{i}}, \\
& \mathbf{k}_{i}=k^{*}\left(\sin \bar{\vartheta}_{i} \cos \varphi_{i} \mathbf{x}_{0}+\sin \bar{\vartheta}_{i} \varphi_{i} \mathbf{y}_{0}+\cos \bar{\vartheta}_{i} \mathbf{z}_{0}\right), \\
& \mathbf{M}_{m}=\mathbf{m}_{m} \mathrm{e}^{i m \varphi} \mathrm{e}^{i k_{z} z-i \omega t}, \\
& \mathbf{N}_{m}=\mathbf{n}_{m} \mathrm{e}^{i m \varphi} \mathrm{e}^{i k_{z} z-i \omega, t}
\end{aligned}
$$

with:

$$
\begin{aligned}
\mathbf{m}_{m} & =i m \frac{Z_{m}\left(k_{\rho} \rho\right)}{\rho} \boldsymbol{\rho}_{0}-k_{\rho} \frac{\partial \rho Z_{m}\left(k_{\rho} \rho\right)}{\partial \rho} \boldsymbol{\varphi}_{0} \\
\mathbf{n}_{m} & =i \frac{k_{z} k_{\rho}}{k} \frac{\partial \rho Z_{m}\left(k_{\rho} \rho\right)}{\partial \rho} \boldsymbol{\rho}_{0}-\frac{m k_{z}}{k} \frac{Z_{m}\left(k_{\rho} \rho\right)}{\rho} \boldsymbol{\varphi}_{0}+ \\
& +\frac{k_{\rho}^{2}}{k} Z_{m}\left(k_{\rho} \rho\right) \mathbf{z}_{0}
\end{aligned}
$$

having indicated, by $k^{*}$, the complex conjugate of the wavenumber $k$ and by $\boldsymbol{\rho}_{0}, \boldsymbol{\varphi}_{0}$ the unit vectors of the cylindrical coordinate system. Considering several parallel cylinders in free space, let us analyze their scattering with the defined incident field, as shown in Fig. 2.

An arbitrarily assigned number $L$ of dielectric cylinders, with relative permittivities $\varepsilon_{j}$, with $j=1, \ldots, N$, infinite length and radii $r_{j}$ in a free-space filled by a lossy medium, in general dissipative, with relative permittivity $\varepsilon_{e}$, relative permeability $\mu_{e}$, and electric conductivity $\sigma_{e}$ is considered. The incident field, as usual, is an elliptically polarized inhomogeneous plane wave. In order to apply Foldy-Lax multiple scattering equations, the external field on the surface of the $q$-th cylinder, also referred to as the exciting field, needs to be taken into consideration. The exciting

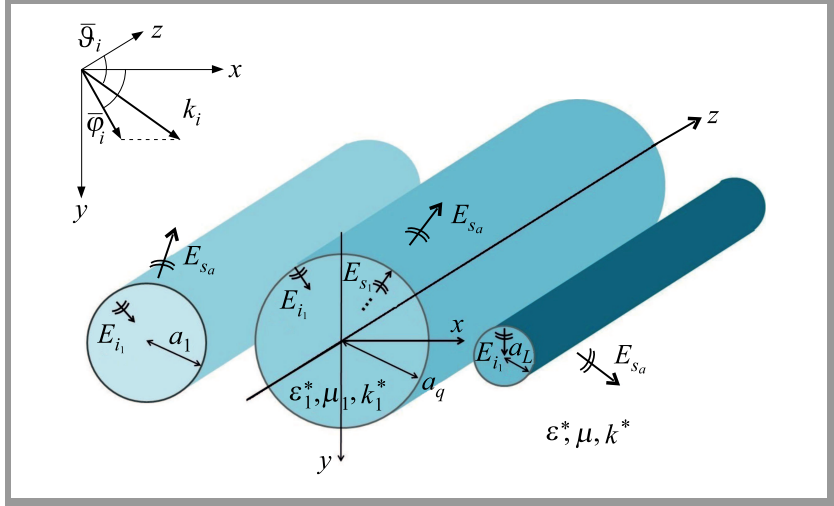

Fig. 2. Depiction of the problem.

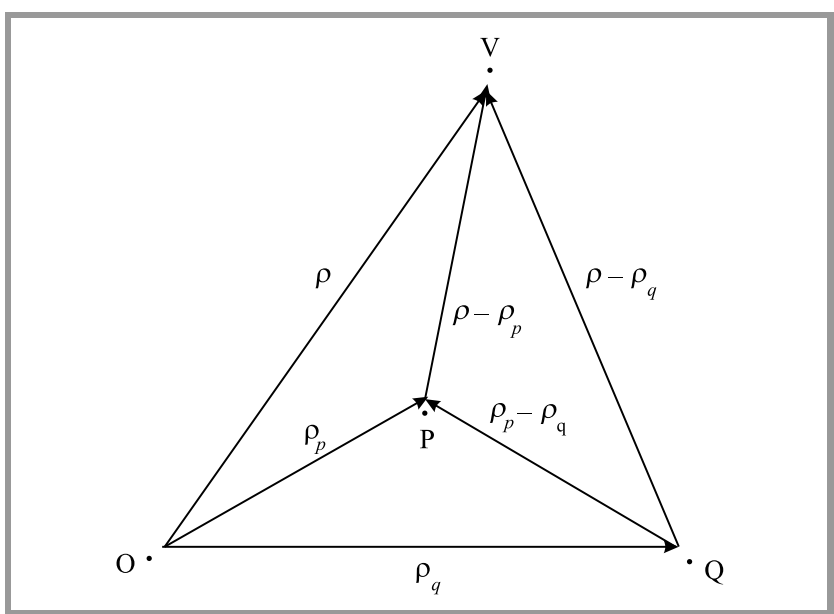

Fig. 3. Given three reference systems $(\mathrm{O}, \mathrm{P}, \mathrm{Q})$, the following figure shows the reciprocal directions of vectors $\boldsymbol{\rho}$ with respect to an external observation point $\mathrm{V}$.

field $\left(\mathbf{E}_{e x}^{q}\right)$ is the superposition of the incident field $\left(\mathbf{E}_{i}\right)$ and all fields scattered by the cylinders $\left(\mathbf{E}_{S}^{p}\right)$, see Fig. 3:

$$
\mathbf{E}_{e x}^{q}=\mathbf{E}_{i}+\sum_{\substack{p=1 \\ p \neq q}}^{L} \mathbf{E}_{s}^{p}
$$

The incident field may be expressed as a function of vector cylindrical harmonics centered on the $q$-th cylinder [36]:

$$
\begin{aligned}
& \mathbf{E}_{i}\left(k \boldsymbol{\rho}_{q}\right)=\left[E_{v i} \boldsymbol{v}+E_{h i} \boldsymbol{h}\right] \mathrm{e}^{i \mathbf{k} \cdot \boldsymbol{\rho}_{q}} \mathrm{e}^{i \mathbf{k} \cdot\left(\boldsymbol{\rho}-\boldsymbol{\rho}_{q}\right)}= \\
= & \sum_{m=-\infty}^{+\infty}\left[\tilde{a}_{m} \mathbf{M}_{m}^{(1)}\left(k, \boldsymbol{\rho}-\boldsymbol{\rho}_{q}\right)+\tilde{b}_{m} \mathbf{N}_{m}^{(1)}\left(k, \boldsymbol{\rho}-\boldsymbol{\rho}_{q}\right)\right],
\end{aligned}
$$

with:

$$
\tilde{a}_{m}=a_{m} \mathrm{e}^{i \mathbf{k} \cdot \boldsymbol{\rho}_{q}}, \quad \tilde{b}_{m}=b_{m} \mathrm{e}^{i \mathbf{k} \cdot \boldsymbol{\rho}_{q}}
$$

with $k$ indicating the wavenumber of the host medium (see Fig. 2) and with $\boldsymbol{\rho}_{q}$ indicating the radial direction centered in the $q$-th cylinder (see Fig. 3 ). The exiting field of the $q$-th cylinder is: 


$$
\begin{aligned}
& \mathbf{E}_{e x}^{q}\left(k \boldsymbol{\rho}_{q}\right)= \\
& \sum_{m=-\infty}^{+\infty}\left[w_{m}^{q} \mathbf{M}_{m}^{(1)}\left(k, \boldsymbol{\rho}-\boldsymbol{\rho}_{q}\right)+v_{m}^{q} \mathbf{N}_{m}^{(1)}\left(k, \boldsymbol{\rho}-\boldsymbol{\rho}_{q}\right)\right],
\end{aligned}
$$

while the scattered electric field from $p \neq q$-th cylinder is:

$$
\begin{aligned}
\mathbf{E}_{s}^{p}\left(k \boldsymbol{\rho}_{p}\right)=\sum_{m^{\prime}=-\infty}^{+\infty} & {\left[T_{m^{\prime}}^{M} w_{m^{\prime}}^{p} \mathbf{M}_{m^{\prime}}^{(3)}\left(k, \boldsymbol{\rho}-\boldsymbol{\rho}_{p}\right)\right.} \\
+ & \left.T_{m^{\prime}}^{N} v_{m^{\prime}}^{p} \mathbf{N}_{m^{\prime}}^{(3)}\left(k, \boldsymbol{\rho}-\boldsymbol{\rho}_{p}\right)\right],
\end{aligned}
$$

with $T_{m^{\prime}}^{M}$ and $T_{m^{\prime}}^{N}$ indicating the scattering coefficients in the dielectric cylinder case, i.e. the T-matrix coefficients [24], [25]:

$$
\begin{aligned}
& T_{m}^{M}=-\frac{J_{m}^{\prime}\left(k_{i \rho} a\right)}{{H^{\prime}}_{m}^{(1)}\left(k_{i \rho} a\right)}, \\
& T_{m}^{N}=-\frac{J_{m}\left(k_{i \rho} a\right)}{H_{m}^{(1)}\left(k_{i \rho} a\right)},
\end{aligned}
$$

while coefficients $w_{m^{\prime}}^{p}$ and $v_{m^{\prime}}^{p}$ represent the unknowns of our problem. By applying the addition theorem to the VCHs function, we obtain:

$$
\begin{aligned}
& M_{m^{\prime}}^{(3)}\left(k, \boldsymbol{\rho}-\boldsymbol{\rho}_{q}\right)=\sum_{m=-\infty}^{+\infty} A_{m m^{\prime}} M_{m}^{(1)}\left(k, \boldsymbol{\rho}-\boldsymbol{\rho}_{q}\right), \\
& N_{m^{\prime}}^{(3)}\left(k, \boldsymbol{\rho}-\boldsymbol{\rho}_{q}\right)=\sum_{m=-\infty}^{+\infty} A_{m m^{\prime}} N_{m}^{(1)}\left(k, \boldsymbol{\rho}-\boldsymbol{\rho}_{q}\right), \\
& M_{m^{\prime}}^{(1)}\left(k, \boldsymbol{\rho}-\boldsymbol{\rho}_{q}\right)=\sum_{m=-\infty}^{+\infty} B_{m m^{\prime}} M_{m}^{(1)}\left(k, \boldsymbol{\rho}-\boldsymbol{\rho}_{q}\right), \\
& N_{m^{\prime}}^{(1)}\left(k, \boldsymbol{\rho}-\boldsymbol{\rho}_{q}\right)=\sum_{m=-\infty}^{+\infty} B_{m m^{\prime}} N_{m}^{(1)}\left(k, \boldsymbol{\rho}-\boldsymbol{\rho}_{q}\right),
\end{aligned}
$$

with:

$$
\begin{aligned}
& A_{m m^{\prime}}=H_{m-m^{\prime}}^{(1)}\left(k\left|\boldsymbol{\rho}_{p}-\boldsymbol{\rho}_{q}\right|\right) \mathrm{e}^{-i\left(m-m^{\prime}\right) \varphi_{p q}}, \\
& B_{m m^{\prime}}=J_{m-m^{\prime}}^{(1)}\left(k\left|\boldsymbol{\rho}_{p}-\boldsymbol{\rho}_{q}\right|\right) \mathrm{e}^{-i\left(m-m^{\prime}\right) \varphi_{p q}} .
\end{aligned}
$$

By replacing all fields inside the FLMSEs and using the orthogonal properties of the VCHs, the following linear system is obtained:

$$
\begin{gathered}
w_{m}^{q}=\tilde{a}_{m}+\sum_{m^{\prime}=-\infty}^{+\infty} \sum_{\substack{p=1 \\
p \neq q}} A_{m m^{\prime}} T_{m^{\prime}}^{M} w_{m^{\prime}}^{p}, \\
v_{m}^{q}=\tilde{b}_{m}+\sum_{m^{\prime}=-\infty}^{+\infty} \sum_{\substack{p=1 \\
p \neq q}} A_{m m^{\prime}} T_{m^{\prime}}^{N} v_{m^{\prime}}^{p} .
\end{gathered}
$$

At this point, the linear system may be solved and coefficients $w_{m}^{q}$ and $v_{m}^{q}$ may be determined. With the field scattered by the $q$-th cylinder, writable as a superposition of VCHs, as:

$$
\mathbf{E}_{s}^{q}=\sum_{m=-\infty}^{+\infty}\left[e_{m}^{q} \mathbf{M}_{m}^{(3)}\left(k, \boldsymbol{\rho}-\boldsymbol{\rho}_{q}\right)+f_{m}^{q} \mathbf{N}_{m}^{(3)}\left(k, \boldsymbol{\rho}-\boldsymbol{\rho}_{q}\right)\right],
$$

the coefficients of the $q$-th cylinder may be written as follows:

$$
\begin{gathered}
e_{m}^{q}=T_{m}^{M} w_{m}^{q}, \\
f_{m}^{q}=T_{m}^{N} v_{m}^{q},
\end{gathered}
$$

and the total scattered field may be obtained:

$$
\mathbf{E}_{s}=\sum_{q=1}^{L} \mathbf{E}_{s}^{q}
$$

\section{Validation and Numerical Results}

Results of the comparison between the formulation and the canonical case of electromagnetic scattering were validated. In this research paper, three infinite lossy dielectric cylinders have been considered with a radius of $a_{1}=a_{2}=a_{3}=0.125 \mathrm{~m}$. The three cylinders are centered at the following $z$ plane coordinates: $C_{1}=[-0.5,0]$, $C_{2}=[0,0]$, and $C_{3}=[0.5,0] \mathrm{m}$. A $1 \mathrm{~V} / \mathrm{m}$ plane wave with a frequency of $300 \mathrm{MHz}$ is impinging on these cylinders.

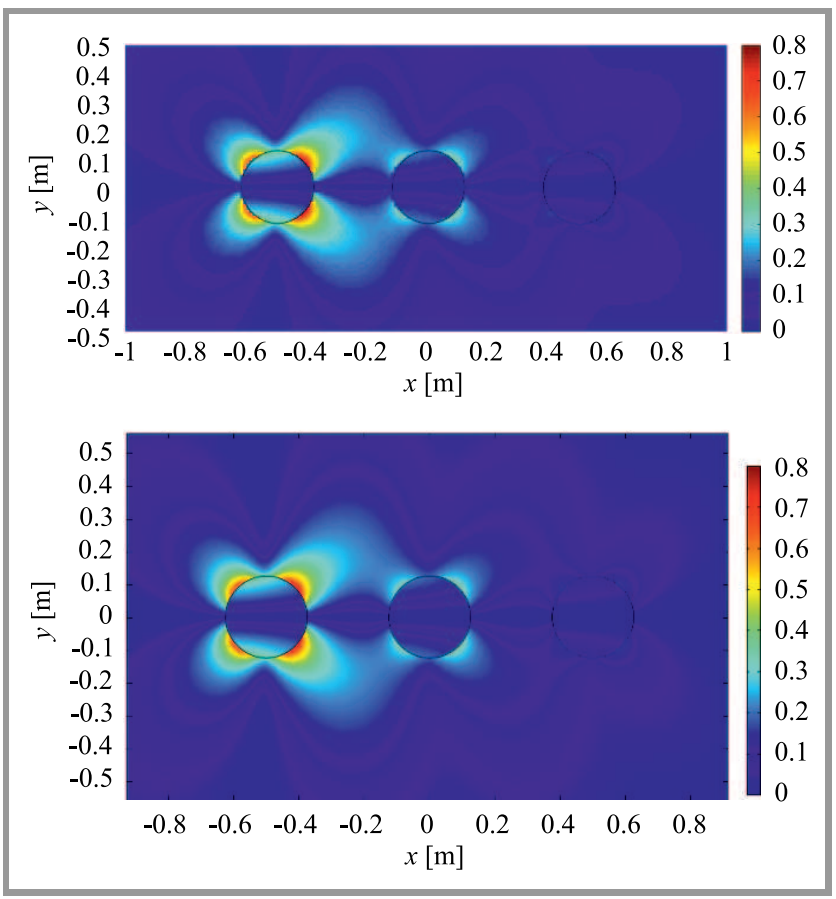

Fig. 4. Comparison of spatial distribution (on the $x-y$ plane) of the absolute value of the $x$ component of the scattered electric field $\left|E_{S_{x}}\right|$. In particular, Matlab (top) and Comsol (bottom) results are shown. (For color pictures see the electronic version of the paper).

In Figs. 4 and 5, results obtained with Matlab and Comsol in the case of $k_{e}=1-i 1 / \mathrm{m}$ for the environment and $k_{c}=2-0.5 i 1 / \mathrm{m}$ for all cylinders (arbitrary parameters) for an incident wave directed at $\vartheta_{i}=\pi / 2$ and $\varphi_{i}=0 \mathrm{rad}$ are displayed. In particular, the absolute values of the $x$ and $y$ components of the scattered electric field (the $z$ component is null) are shown. As noted, the results are perfectly compatible. 


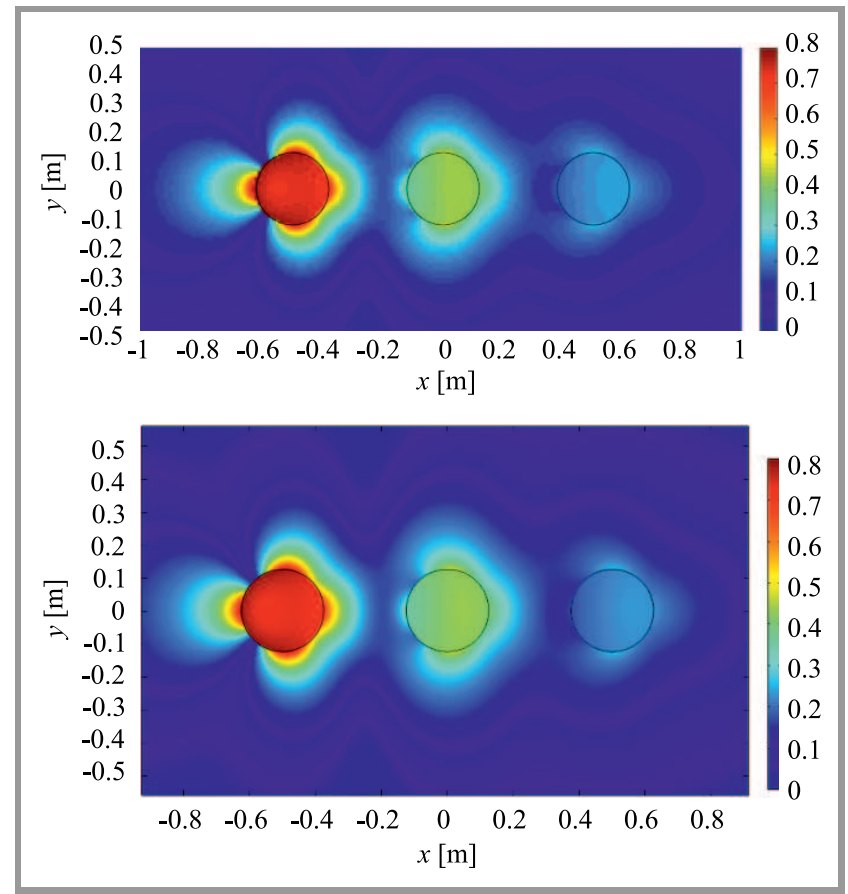

Fig. 5. Comparison of spatial distribution (on the $x-y$ plane) of the absolute value of the $y$ component of the scattered electric field $\left|E_{S_{y}}\right|$. In particular, Matlab (top) and Comsol (bottom) results are shown.

Subsequently (see Fig. 6), the most general case of an inhomogeneous plane wave with $\vartheta$ complex value was shown. In particular, we have considered the following values for the complex angle: $\vartheta=\pi / 6(1+0.5 i) \mathrm{rad}$ and $\varphi=\pi / 4$ rad (arbitrary parameters). The remaining parameters have remained the same as in the previous case. To obtain these

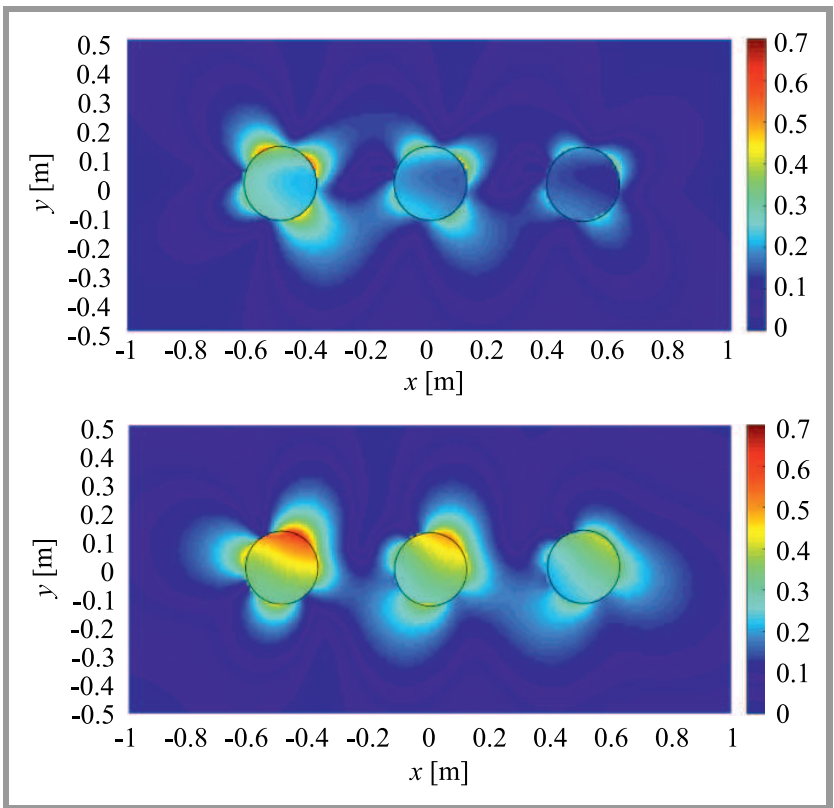

Fig. 6. Spatial distribution of the absolute value (on the $x-y$ plane) of the $x$ (top) and $y$ (bottom) components of the scattered electric field $\left|E_{S_{x}}\right|,\left|E_{S_{y}}\right|$ obtained with Matlab. results, $N=5$ was used as the number of terms for the VCHs series [38].

In Fig. 6, the contribution of the complex angle is highlighted by the direction of the scattered wave which creates an attenuation effect spreading in the same direction.

\section{Conclusions}

An accurate method allowing to express an inhomogeneous, elliptically polarized plane wave in terms of vectoral cylinder harmonics, needed to solve the problem of multi-scattering generated by an ensemble of cylinders, is presented. Determination of the expansion coefficients and application of the so-called Foldy-Lay equations required to use the complex-angle formalism contribute to the determination of the solution to the electromagnetic problem. A light and elegant formalism has been reached thanks to such an approach. The procedure was validated with some numerical results as well as with comparisons performed via simulations in the COMSOL environment. In particular, the results were compared with regard to scattering created by three lossy dielectric cylinders of a circular cross-section and infinite length. Perfect compatibility was reached in all scenarios. The cylindrical harmonics defined with the complex angle share the same properties as simple cylindrical harmonics, thanks to the minimal invasiveness of the formalism.

\section{References}

[1] G. Mie, "Beitrage zur Optik truber medien, speziell kolloidaler metallosungen", Ann. Physik, vol. 25, 1908 (DOI: 10.1002//andp.19083300302) [in German].

[2] J. J. Bowman, T. B. A. Senior, and P. L. E. Uslenghi, Electromagnetic and Acoustic Scattering by Simple Shapes. Amsterdam: NorthHolland Publishing Company, 1969 (ISBN: 97807200401523).

[3] J. R. Parry, "Electromagnetic scattering from cylinders of arbitrary cross-section in a conductive half-space", Geophysics, vol. 36, no. 1, pp. 1-217, 1971 (DOI: 10.1190/1.1440165).

[4] P. Latimer, "Light scattering by ellipsoids", J. of Colloid and Interf. Sci., vol. 53, no. 1, pp. 102-109, 1975 (DOI: 10.1016/0021-9797(75)90039-9).

[5] T. Rother, Electromagnetic Wave Scattering on Nonspherical Particles. New York: Springer, 2009 (ISBN: 9783642007033).

[6] C. A. Balanis, Advanced Engineering Electromagnetics, 2nd ed. Wiley, 2012 (ISBN: 9780470589489).

[7] R. Borghi, F. Gori, M. Santarsiero, F. Frezza, and G. Schettini, "Plane-wave scattering by a perfectly conducting circular cylinder near a plane surface: cylindrical-wave approach", J. of the Optic. Soc. of America A, vol. 13, no. 3, pp. 483-493, 1996 (DOI: 10.1364/JOSAA.13.000483).

[8] T. Wriedt and A. Doicu, "Light scattering from a particle on or near a surface", Optics Commun., vol. 152, no. 4-6, pp. 376-384, 1998 (DOI: 10.1016/S0030-4018(98)00099-6).

[9] S.-C. Lee and A. Grzesik, "Light scattering by closely spaced parallel cylinders embedded in a semi-infinite dielectric medium", J. of the Optic. Soc. of America A, vol. 15, no. 1, pp. 163-173, 1998 (DOI: 10.1364/JOSAA.15.000163).

[10] A. Doicu, T. Wriedt, and Y. A. Eremin, Light Scattering by Systems of Particles. New York: Springer, 2006 (ISBN: 9783540336969). 
[11] F. Frezza, F. Mangini, L. Pajewski, G. Schettini, and N. Tedeschi, "Spectral domain method for the electromagnetic scattering by a buried sphere", J. of the Optic. Soc. of America A, vol. 30, no. 4, pp. 783-790, 2013 (DOI: 10.1364/JOSAA.30.000783).

[12] F. Frezza, F. Mangini, and N. Tedeschi, "Electromagnetic scattering by two concentric spheres buried in a stratified material", $J$. of the Optic. Soc. of America A, vol. 32, no. 2, pp. 277-286, 2015 (DOI: $10.1364 /$ JOSAA.32.000277).

[13] F. Frezza and F. Mangini, "Electromagnetic scattering by a buried sphere in a lossy medium of an inhomogeneous plane wave at arbitrary incidence: Spectral-domain method", J. Optic. Soc. of America $A$, vol. 33, no. 5, pp. 947-953, 2016 (DOI: 10.1364/JOSAA.33.000947).

[14] F. Mangini, N. Tedeschi, F. Frezza, and A. Sihvola, "Homogenization of a multilayer sphere as a radial uniaxial sphere: features and limits", J. Electrom. Waves Appl., vol. 28, no. 8, pp. 916-931, 2014 (DOI: $10.1080 / 09205071.2014 .896480)$.

[15] F. Mangini, N. Tedeschi, F. Frezza, and A. Sihvola, "Electromagnetic scattering of an inhomogeneous elliptically polarized plane wave by a multilayered sphere", J. Electrom. Waves Appl., vol. 30, no. 4, pp. 492-504, 2016 (DOI: $10.1080 / 09205071.2015 .1121842)$.

[16] F. Frezza and F. Mangini, "Electromagnetic scattering of an inhomogeneous elliptically polarized plane wave by a multilayered sphere", J. Electrom. Waves Appl., vol. 30, no. 4, pp. 492-504, 2016 (DOI: 10.1080/09205071.2015.1121842).

[17] F. Mangini and N. Tedeschi, "Scattering of an electromagnetic plane wave by a sphere embedded in a cylinder", J. of the Optic. Soc. of America A, vol. 34, no. 5, pp. 760-769, 2017 (DOI: $10.1364 /$ JOSAA.34.000760).

[18] S. N. Samaddar, "Scattering of plane waves from an infinitely long cylinder of anisotropic materials at oblique incidence with an application to an electronic scanning antenna", Appl. Scient. Res. B, vol. 10, pp. 385-411, 1962 (DOI: 10.1007/BF02923451).

[19] X. B. Wu and W. Ren, "Wave-function solution of plane-wave scattering by an anisotropic circular cylinder", Microw. and Opt. Technol. Lett., vol. 8, no. 1, pp. 39-42, 1995 (DOI: $10.1002 / \mathrm{mop} .4650080114$ )

[20] S.-C. Mao and Z.-S. Wu, "Scattering by an infinite homogeneous anisotropic elliptic cylinder in terms of Mathieu functions and Fourier series", J. of the Optic. Soc. of America A, vol. 25, no. 12, pp. 2925-2931, 2008 (DOI: 10.1364/JOSAA.25.002925).

[21] F. Frezza, F. Mangini, M. Muzi, and E. Stoja, "In silico validation procedure for cell volume fraction estimation through dielectric spectroscopy", J. of Biolog. Physics, vol. 41, no. 3, pp. 223-234, 2015 (DOI: 10.1007/s10867-014-9374-8).

[22] G. Videen, "Light scattering from a particle on or near a perfectly conducting surface", Optics Commun., vol. 115, no. 1-2, pp. 1-7, 1995 (DOI: 10.1016/0030-4018(94)00668-K).

[23] M. I. Mishchenko, L. D. Travis, and A. A. Lacis, Scattering, Absorption, and Emission of Light by Small Particles. Cambridge: Cambridge University Press, 2002 (ISBN: 9780521782524).

[24] L. Tsang and J. A. Kong, Scattering of Electromagnetic Waves: Theory and Applications. New York: Wiley, 2000 (ISBN: 9780471224280).

[25] L. Tsang, J. A. Kong, K.-H. Ding, and C. O. Ao, Scattering of Electromagnetic Waves: Numerical Simulations. New York: Wiley, 2000 (ISBN: 9780471388005).

[26] E. I. Ivlev, "Structure and properties of inhomogeneous waves", J. of Modern Optics, vol. 34, no. 12, pp. 1559-1569, 1987 (DOI: 10.1080/09500348714551491).

[27] E. I. Ivlev, "The scattering of inhomogeneous electromagnetic waves by a cylinder", J. of Modern Optics, vol. 39, no. 3, pp. 499-507, 1992 (DOI: 10.1080/09500349214550491).

[28] R. B. Adler, L. J. Chu, and R. M. Fano, Electromagnetic Energy Transmission and Radiation. MIT Press Classics, 1968 (ISBN: 9780262511407).

[29] F. Frezza and F. Mangini, "Vectorial spherical-harmonics representation of an inhomogeneous elliptically polarized plane wave", J. of the Optic. Soc. of America A, vol. 32, no. 7, pp. 1379-1383, 2015 (DOI: 10.1364/JOSAA.32.001379).
[30] C. F. Bohren and D. R. Huffman, Absorption and Scattering of Light by Small Particles. Wiley, 1998 (ISBN: 9780471293408).

[31] J. A. Stratton, Electromagnetic Theory. New York and London: McGraw-Hill, 1941 (ISBN: 9780070621503) [Online]. Available: https://archive.org/details/electromagnetict $031016 \mathrm{mbp} /$ page/n5

[32] M. Kerker, The Scattering of Light. New York, San Francisco and London: Academic Press, 1969 (ISBN: 9781483191744).

[33] M. Born and E. Wolf, Principles of Optics, 7th ed. London: Cambridge Press, 1999 (ISBN: 9781139644181).

[34] L. Tsang, J. A. Kong, and K. H. Ding, Scattering of Electromagnetic Waves: Theory and Applications. New York: Wiley, 2000 (ISBN: 9780471224280).

[35] J. G. Van Bladel, Electromagnetic Fields, 2nd ed. New Jersey: Wiley, 2007 (ISBN: 9780471263883).

[36] F. Frezza, F. Mangini, and N. Tedeschi, "Introduction to electromagnetic scattering: tutorial", J. of the Optic. Soc. of America A, vol. 35, no. 1, pp. 163-173, 2018 (doi: 10.1364/JOSAA.35.000163).

[37] F. Mangini, L. Dinia, and F. Frezza, "Electromagnetic scattering by a cylinder in a lossy medium of an inhomogeneous elliptically polarized plane wave", J. of Telecommun. and Inform. Technol., vol. 4, pp. 36-42, 2019 (DOI: 10.26636/jtit.2019.135819).

[38] S. Batool, F. Frezza, F. Mangini, and F. Yu-Lin Xu, "Scattering from multiple PEC sphere using translation addition theorems for spherical vector wave function", J. of Quantit. Spectros. \& Radia. Transfer, vol. 248, no. 17, pp. 1-6, 2020 (DOI: 10.1016/j.jqsrt.2020.106905).

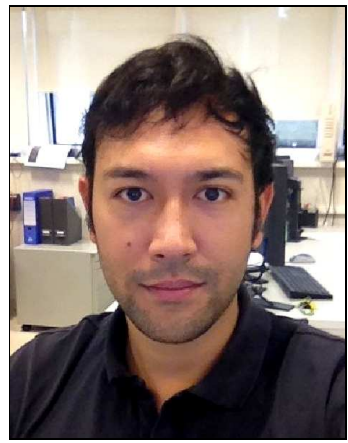

Fabio Mangini received his B.Sc. in Clinical Engineering in and M.Sc. in Biomedical Engineering "cum laude" from "La Sapienza" University of Rome, Italy in 2005 and 2008, respectively. He earned his $\mathrm{Ph} . \mathrm{D}$. in Electromagnetism from the Department of Information Engineering, Electronics and Telecommunications of the same University in 2014. In June 2014 and in May 2015, he won the "Young Scientist Award" from URSI (International Union of Radio Science). In January 2017, he won the "PhD ITalents" prize and in October 2018 he won the "Marabelli prize". Between 2009 and 2015 he worked at the Laboratory of Electromagnetic Fields II at "La Sapienza" University of Rome. His research activities focus on guiding structures, numerical methods, theoretical scattering models, optical propagation, anisotropic media, metamaterials, biomedical applications, and culturalheritage applications. Since April 2019, he has been working, as a researcher, at the Electromagnetic Fields and Photonics group at the University of Brescia.

(iD) https://orcid.org/0000-0001-5474-8952

E-mail: fabio.mangini@unibs.it

Department of Information Engineering (DII)

University of Brescia

Via Branze 38

25123 Brescia, Italy 


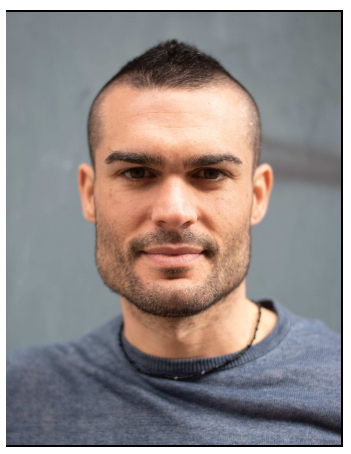

Lorenzo Dinia graduated with a 4.0 GPA, earning his M.Sc. in Biomedical Engineering in Rome. In 2014, he earned a second M.Sc. in Industrial Engineering from NYU Tandon School of Engineering. In February 2019, he completed a Ph.D. program in Mathematical Models for Engineering Electromagnetics and Nanosciences, curriculum in Electromagnetics, at Sapienza University of Rome. His these focused on the fiber Bragg grating sensor and its applications, mainly related to the conservation of works of art. He has three years of experience as a medical equipment Maintenance Manager at two major hospitals in Rome. He was responsible for a team of technicians tasked with coordinating corrective and preventive maintenance and performing quality inspections. During his professional career, he has held different engineering roles in USA. In 2015, he was a Process Technical Engineer at a manufacturing company in Brooklyn. In 2016, he worked as a Field Service Engineer at a packaging company in New Jersey. Currently, he is working as a Quality Engineer II at a supplier of medical devices.

(iD) https://orcid.org/0000-0001-5725-0930

E-mail: lorenzo.dinia@gmail.com

Department of Information Engineering, Electronics and Telecommunications (DIET)

"La Sapienza" University of Rome

Via Eudossiana 18

00184 Rome, Italy

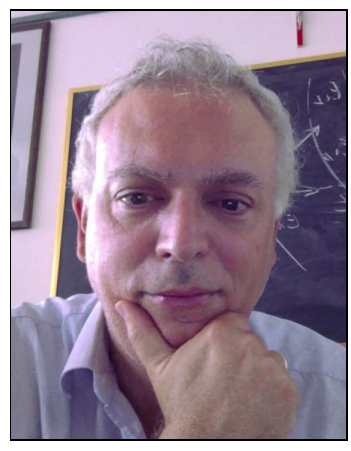

Fabrizio Frezza received his "Laurea" (degree) "cum laude" in Electronic Engineering in 1986 and his Ph.D. in Applied Electromagnetics and Electrophysical Sciences in 1991, both from "La Sapienza" University of Rome. In 1986, he joined the Department of Electronics of the same University, where he held the position of a Researcher from 1990 to 1998, a temporary Professor of Electromagnetic Fields from 1994 to 1998, an Associate Professor from 1998 to 2004, and he has been a Full Professor of Electromagnetic Fields since 2005. His research activities focus on electromagnetic waveguides, antennas and resonators, mathematical and numerical methods, electromagnetic scattering, optics, free electromagnetic propagation, thermonuclear-plasma heating, anisotropic media, artificial materials and metamaterials, plasmonics, biomedical applications, cultural-heritage and environment applications, artificial-intelligence applications in electromagnetic sensing and diagnostics, magnetic-resonance applications, electrical transmission lines, electromagnetic compatibility, spectroscopy, terahertz applications, technological transfer, history of science and technology.

(iD) https://orcid.org/0000-0001-9457-7617

E-mail: fabrizio.frezza@uniroma1.it

Department of Information Engineering, Electronics and Telecommunications (DIET)

"La Sapienza" University of Rome

Via Eudossiana 18

00184 Rome, Italy 